\title{
Exercise Testing in Mitral Regurgitation
}

\section{Raluca Dulgheru ${ }^{a}$, Stella Marchetta ${ }^{a}$, Tadafumi Sugimoto ${ }^{a}$, Yun Yun Go ${ }^{b}$, Alexandra Girbea ${ }^{a}$, Cécile Oury ${ }^{c}$, Patrizio Lancellotti ${ }^{a, c}, d, *$}

${ }^{a}$ University of Liège Hospital, Department of Cardiology, Heart Valve Clinic, Liège, Belgium

${ }^{b}$ National Heart Research Institute Singapore, National Heart Centre Singapore, Singapore

${ }^{\mathrm{C}}$ GIGA Cardiovascular Sciences, University Hospital Sart Tilman, Liège, Belgium

${ }^{\mathrm{d} G r u p p o ~ V i l l a ~ M a r i a ~ C a r e ~ a n d ~ R e s e a r c h, ~ A n t h e a ~ H o s p i t a l, ~ B a r i, ~ I t a l y ~}$

\section{A R T I C L E I N F O}

\section{Keywords:}

Primary mitral regurgitation

Secondary mitral regurgitation

Exercise testing

Exercise stress echocardiography

Valvular heart disease

\begin{abstract}
A B S T R A C T
Mitral regurgitation (MR) is the second most common valvular heart disease referred for corrective surgery. Diagnostic and management dilemmas are not uncommon when dealing with MR patients. Exercise testing plays an important role in sorting out some of these clinical challenges. In primary asymptomatic MR, exercise testing allows symptom assessment, confident link of symptoms to valve disease severity, safe deferral of surgery for the next 1-year in patients with preserved exercise capacity, insights into the mechanism of exercise-induced dyspnea and helps in individual risk stratification. Moreover, exercise testing in the form of exercise stress echocardiography is also useful in the evaluation of patients with secondary ischemic MR for risk stratification as well as for the detection of patients with moderate ischemic MR in whom mitral valve repair at the time of surgical revascularization may add benefit.
\end{abstract}

(C) 2017 Elsevier Inc. All rights reserved.

\section{Contents}

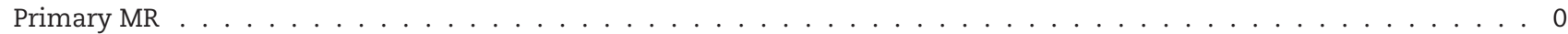

The complex tale of exercise testing - why do we even bother? . . . . . . . . . . . . . . . . . . . . . . 0

Linking symptoms to $\mathrm{MR} \ldots \ldots \ldots \ldots \ldots$

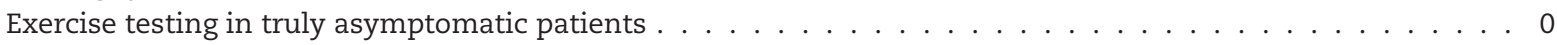

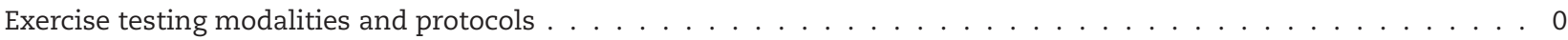

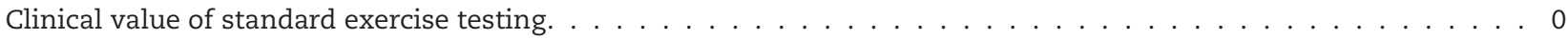

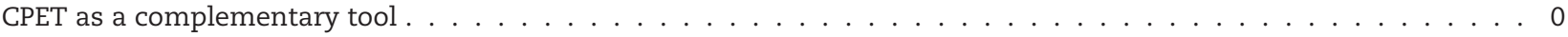

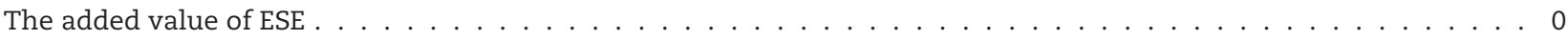

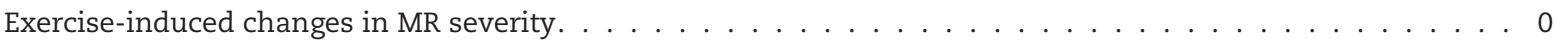

Exercise-induced $\mathrm{PH} \ldots \ldots \ldots \ldots \ldots$

LV systolic function reserve to exercise . . . . . . . . . . . . . . . . . . . . . . . 0

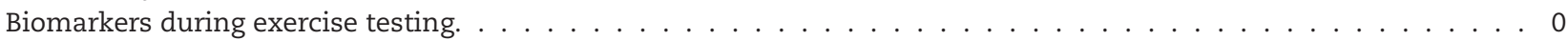

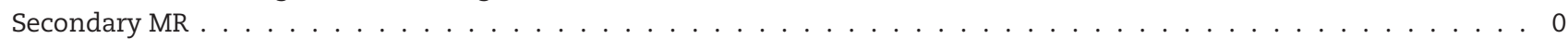

Statement of conflict of interest: see page XX.

* Address reprint requests to Patrizio Lancellotti, MD, PhD, Domaine Universitaire du Sart Tilman, Batiment B35, Department of Cardiology, University Hospital, Université de Liège, CHU du Sart Tilman, 4000 Liège, Belgium.

E-mail address: plancellotti@chu.ulg.ac.be (P. Lancellotti). 
Role of ESE. . . . . . . . . . . . . . . . . . . . . . . . 0

Conclusions . . . . . . . . . . . . . . . . . . . . . 0

Statement of conflict of interest . . . . . . . . . . . . . . . . . . . . 0

References. . . . . . . . . . . . . . . . . . . . . 0
Abbreviations and Acronyms

ACC $=$ American College

Cardiology

$\mathrm{AF}=$ Atrial fibrillation

$\mathrm{BNP}=$ Brain natriuretic peptide

$\mathrm{BP}=$ Blood pressure

CPET $=$ Cardiopulmonary exercise testing

CRT $=$ Cardiac resynchronization therapy fice area

ESC = European Society of Cardiology echocardiography

GLS = Global longitudinal strain

$\mathrm{HF}=$ Heart failure

LA = Left atrial

$\mathrm{LV}=$ Left ventricular

LVEF = Left ventricular ejection fraction

MR = Mitral regurgitation

MV = Mitral valve

$\mathrm{PAP}=$ Pulmonary artery pressure

$\mathrm{PH}=$ Pulmonary hypertension

$\mathrm{RV}=$ Right ventricular

RVSP = Right ventricular systolic pressure

SBP $=$ Systolic blood pressure

SMR = Secondary mitral

regurgitation

SPAP = Systolic pulmonary artery pressure

VHD = Valvular heart disease

VO2 = Oxygen consumption
EROA = Effective regurgitant ori-

ESE $=$ Exercise stress

HRR = heart rate recovery

Mitral valve (MV) regurgitation (MR) is the second most common valvular heart disease (VHD) referred for corrective surgery. ${ }^{1}$ It is also the valvulopathy that poses the most clinical dilemmas. Conventionally, MR is classified in primary or secondary MR. Rarely, mixed forms of MR may be encountered. In primary MR, it is the MV apparatus (i.e. leaflets, annulus, chordae or combination) that carries the disease. The left ventricle (LV), left atrium (LA) and pulmonary vascular bed suffer from the consequences of the chronic volume overload imposed by the MR. To the opposite, secondary MR is the result of a diseased LV, while MV apparatus is intact. It is the MV that suffers from the consequences of the LV dysfunction and dilatation by becoming incompetent. In turn, secondary MR is also a contributor to LV dilatation and dysfunction. The cardinal symptom of $\mathrm{MR}$ is exercise-dyspnea or,

in the advanced form, signs and symptoms of overt heart failure. However, in developed countries, due to the high accessibility to the medical system, the diagnosis is usually made earlier in the disease's course, when patients are still asymptomatic. For secondary MR and patients with asymptomatic primary $M R$, the full clinical impact of the disease is underestimated whenever work-up ends with a simple transthoracic echocardiography (TTE). To this regard and for their risk stratification capabilities exercise testing is advocated by the current guidelines on the management of VHD. ${ }^{2,3}$ This review will address the role of exercise testing in both primary and secondary MR.

\section{Primary MR}

The complex tale of exercise testing - why do we even bother?

When present and if uncorrected, MR impacts patient's survival. ${ }^{4}$ Surgery and, more recently, transcatheter therapies (for high surgical risk patients) are the only available treatments that improve patient's outcome., ${ }^{5,6}$ Based on the current evidence, surgery is recommended (Class I) in patients with severe MR when they are symptomatic (but have a LV ejection fraction (LVEF) $>30 \%$ ) or have overt LV systolic dysfunction (i.e. LVEF $\leq 60 \%$ but $>30 \%$ or an end-systolic LV diameter $\geq 45 \mathrm{~mm}$ (for European Society of Cardiology ESC)/ $\geq 40 \mathrm{~mm}$ [for American College of Cardiology (ACC)/American Heart Association (AHA)]). ${ }^{2,3}$ Although, these indications for surgery appear crystal clear, in clinical practice things may get complicated.

\section{Linking symptoms to $M R$}

Since surgery is indicated once the patient becomes symptomatic, identifying those who are truly asymptomatic is fundamental. Exercise stress testing is an objective way of confirming the absence of symptoms and to define one's true functional capacity, compared against age-gender predicted standards. Not infrequently, patients adapt their daily activities in order to avoid symptoms. However, exercise-induced dyspnea as presenting symptom may be subjective and difficult to objectively link to valve disease itself, since dyspnea may have multiple causes (deconditioning, obesity, pulmonary disease, etc.). To this regard, exercise stress echocardiography (ESE) may be used as a rewarding modality to assess the true hemodynamic consequences of MR and to match symptoms with the severity of cardiac involvement secondary to MR.

\section{Exercise testing in truly asymptomatic patients}

The management of asymptomatic patients with severe primary MR and no LV enlargement or dysfunction remains a source of debate. The recent ACC/AHA guidelines, however, advocate MV repair (Class IIa) in these patients when there is $>95 \%$ likelihood of a successful and durable repair without residual $\mathrm{MR}$, and a $<1 \%$ expected mortality rate in a center of excellence. $^{3,7}$ Similar reasonable indication exists in the ESC guidelines but in patients with flail MV or significantly dilated LA(IIa). ${ }^{2}$ Other reasonable triggers for surgery (class IIa) 
Table 1 - Summary of different modalities and protocols used in exercise testing.

\begin{tabular}{llll} 
Modality & Protocol & Associated Modality & Timing of Imaging \\
\hline Treadmill & Bruce & Echocardiography & Post-exercise \\
Upstanding bicycle & Modified Bruce & Pulmonary gas exchange analysis (CPET) & Post-exercise \\
Semi-supine bicycle & Naughton & Echocardiography and pulmonary gas exchange analysis (CPET) & During exercise
\end{tabular}

include increased systolic pulmonary artery pressure (SPAP $>50 \mathrm{~mm} \mathrm{Hg}$ ), or development of atrial fibrillation (AF).$^{8-10}$ So, since prophylactic surgery is not unanimously contemplated in all asymptomatic patients with severe MR, patient's management is often dictated by individual risk stratification. ESE provides a series of prognostic features that could help clinical decision-making; early guidance to mitral valve intervention, closer follow-up, or safe watchful waiting. ${ }^{11-19}$

\section{Exercise testing modalities and protocols}

ESE can be performed using either a treadmill or bicycle ergometer, with a symptom-limited protocol in which the workload is gradually increased in stages (Table 1). The most common treadmill protocol used is the Bruce (or modified) protocol, in which the expected exercise level for a given age and sex can be expressed as functional aerobic capacity. Image acquisition takes place rapidly after exercise cessation and focuses first on regional wall motion (hence LV regional and global systolic function) and right ventricular (RV) systolic pressure (RVSP). ${ }^{17,18}$ When compared with cycle ergometer, treadmill exercise has the advantage of reaching higher workloads and heart rates. Semi-supine bicycle exercise is usually the preferred approach because it allows the assessment of multiple stress parameters during each step of exercise testing (Figs. 1-3). At peak exercise, focus is set on the LV systolic function, MR severity, E/e' (an estimate of LV filling pressure), RVSP and RV function (Fig. 1). ${ }^{20}$ The patient pedals at a constant cadence against an increasing workload, which starts at $25 \mathrm{~W}$ (50 W for younger patients) with increments of 25 watts every 2 or $3 \mathrm{~min}$. Adequate bicycle stress testing requires the patient's cooperation and coordination to perform the pedaling action and this may be a disadvantage. ${ }^{20}$ Appearance of symptoms should be assessed regularly, and blood pressure (BP), heart rate and a 12-lead ECG should be monitored continuously during the examination. Criteria for interruption are the target heart rate achievement, or onset of typical chest pain, $\geq 2 \mathrm{~mm}$ horizontal or downsloping ST segment depression, limiting breathlessness, dizziness, hypotension (systolic BP/SBP drop below baseline), complex ventricular arrhythmia and muscular exhaustion. Of note, cardiopulmonary exercise testing (CPET) with measurement of gas exchange, which has the advantage of providing quantitative evaluation of maximal exercise capacity, can also be useful in patients with primary MR. $^{21}$ CPET can be combined with echocardiographic evaluation.

\section{Clinical value of standard exercise testing}

$\mathrm{BP}$ response to exercise is clinically meaningful and easy to access. A drop in SBP during exercise may reflect the time-point during exercise testing at which severe MR is compromising the ability of the LV to increase cardiac output. In the light of recent research, treadmill exercise may be useful to identify a subset of asymptomatic patients with severe primary MR in whom conservative treatment may be initially deferred. ${ }^{17,18}$ In fact, in case of preserved exercise capacity, delaying MV surgery by $\geq 1$ year did not adversely affect outcomes. ${ }^{18}$ Conversely, patients achieving $<85 \%$ of predicted metabolic equivalents which may be observed in up to $30 \%$, or those with an abnormal heart rate recovery (HRR; $<18$ beat drop in heart rate over $1 \mathrm{~min}$ in recovery; probably a marker of impaired vagal activity) have an increased risk of events (all-cause mortality, myocardial infarction, stroke, AF or progression to congestive heart failure/HF), which might be used as incentive findings for referring patients to surgery. In daily practice, the clinical usefulness of exercise capacity assessment may be hampered by several confounding factors since it is the result of the integrated function of the cardiovascular, respiratory and musculoskeletal systems. Therefore, once a patient with altered exercise capacity is detected, the next step is to corroborate it with MR consequences.

\section{CPET as a complementary tool}

MR may negatively affect exercise capacity through a reduction of cardiac output and its resulting retrograde pressure elevation in the pulmonary venous systems. The interest of CPET in patients with severe asymptomatic primary MR results from its ability to assess their pulmonary hemodynamic status (ventilatory efficiency), and their aerobic capacity (cardiac output adaptation to exercise). Peak oxygen consumption (peak VO2) may be considered as a marker of the degree to which primary MR may compromise cardiac output and therefore aerobic capacity. Recent studies have shown that age-gender predicted VO2 varies widely in these patients with severe asymptomatic primary MR. ${ }^{19}$ Up to $20 \%$ of them actually have reduced functional capacity $(\leq 84 \%$ age-gender expected peak VO2), which related to E/e', lower stroke volume, the presence of AF, but not to the severity of MR. In this study, reduced functional capacity (i.e. peak VO2 $\leq 84 \%$ age-gender predicted peak VO2) was an independent marker of higher mid-term risk of clinical events (death, HF or new severe symptoms, new atrial arrhythmia) or surgery. ${ }^{19}$ Interestingly, in patients with asymptomatic moderate to severe primary MR, exercise pulmonary hypertension (PH), a predictor of symptom onset, is an independent echocardiographic determinant of peak VO2 and VE/VCOE slope, an estimate of ventilator efficiency. ${ }^{21,22}$ In practice, although of interest, especially for longitudinal monitoring, CPET is rarely used in patients with MR. 


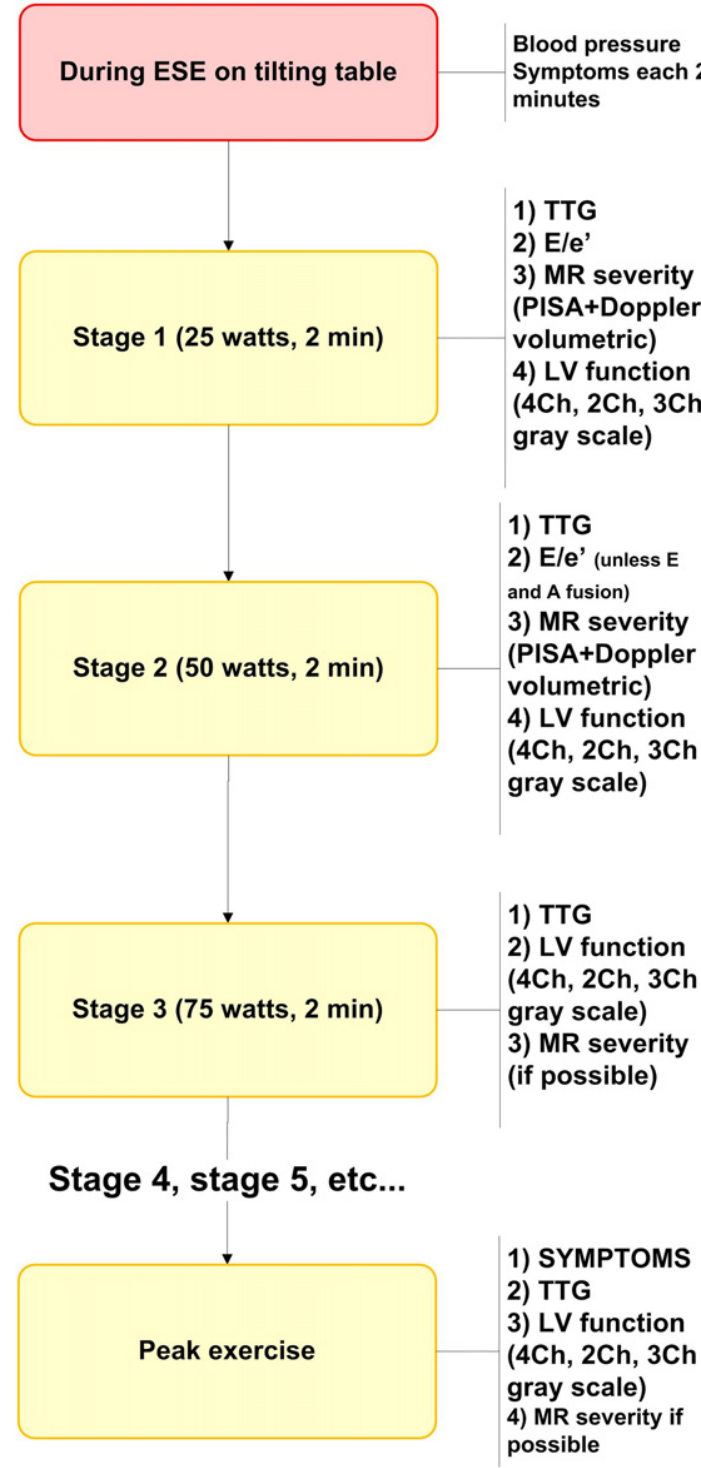

Fig. 1 - Flow-chart on how to perform an exercise stress echocardiography on a semi-supine tilting table. ESE-exercise stress echocardiography; TTG-transtricuspid systolic pressure gradient; E/e'-ratio between early diastolic velocity at the mitral leaflet's tips to early diastolic velocity by TDI at the level of the medial mitral annulus; MR-mitral regurgitation; LV-left ventricle; $4 \mathrm{Ch}$-apical four chambers view; $2 \mathrm{Ch}$-apical two chambers view; $3 \mathrm{Ch}$-apical three chambers view.

\section{The added value of ESE}

In patients with primary MR, ESE, as any other exercise test, may be used to provoke symptoms. SBP drop and abnormal HRR may also be detected. However, in our experience, ESE has a remarkable added value in the understanding of hemodynamics, linking symptoms to changing hemodynamics, risk stratification, and ultimately management of patients with primary MR. Although there is less evidence available, the test seems also reasonable in symptomatic patients with at least moderate MR. ${ }^{12,15}$ The increase in MR severity ( $\geq 1$ grade), ${ }^{12,23}$ exercise induced $\mathrm{PH}$ (exercise systolic pulmonary artery pressure or SPAP $\geq 60 \mathrm{~mm} \mathrm{Hg}),{ }^{15,16,24}$ the absence of LV contractile reserve $(<5 \%$ increase in $\mathrm{EF}$ or $<-2 \%$ increment in global longitudinal strain (GLS)), ${ }^{11,25}$ and failure of the right ventricle to cope with the excessively high pulmonary artery pressure (PAP) during exercise (quantified by tricuspid annular plane systolic excursion $<18 \mathrm{~mm})^{24}$ are all parameters of poor prognosis in asymptomatic patients with severe primary MR. Their systematic assessment during ESE is recommended, whenever possible.

\section{Exercise-induced changes in MR severity}

Primary MR may be a highly dynamic entity. ${ }^{12,26}$ In chronic asymptomatic primary MR, marked exercise-induced augmentation in MR severity (Effective Regurgitant Orifice Area or EROA $\geq 10 \mathrm{~mm}^{2}$ and regurgitant volume $\geq 15 \mathrm{ml}$ ) is present in $1 / 3$ of patients and unrelated to resting MR severity. ${ }^{12}$ The magnitude of this augmentation is correlated with exercise-induced variation in SPAP and reduced symptom-free survival. ${ }^{12,27}$

\section{Exercise-induced $\mathrm{PH}$}

In severe primary MR, progressive increase in LV filling pressures and LA pressure leads to a backward passive rise in pressure in the pulmonary veins and to post-capillary $\mathrm{PH}$. When present at rest, $\mathrm{PH}$ is a marker of poor outcome and surgery is advised (class IIa indication in both guidelines). ${ }^{2,3}$ However, in truly asymptomatic patients, $\mathrm{PH}$ at rest is a seldom finding. Conversely, exercise $\mathrm{PH}$ is more common (up to $46 \%$ in one study) in this group of asymptomatic patients, ${ }^{27}$ even when no $\mathrm{PH}$ is noted at rest. ${ }^{16,27}$ Patients who developed exercise $\mathrm{PH}$ and stop exercise for dyspnea have higher increase in regurgitant volumes and larger increase in EROA with exercise. ${ }^{27}$ Hence, at least in part, the increase in MR severity with exercise may be reflected by the increase in PAP (Fig. 2). Also, exercise PH predicts symptoms onset. ${ }^{27}$ A recent study showed that at 3-year follow-up, only $20 \%$ of the patients with exercise $\mathrm{PH}$ at baseline remained free of symptoms. ${ }^{24,27}$ Exercise $\mathrm{PH}$ is also associated with a higher rate of cardiac death, HF hospitalization, stroke or AF after MV surgery. ${ }^{13,27} \mathrm{PH}$ at exercise (>60 mm Hg, class IIb) can also be considered as a trigger for MV repair in low risk patients. ${ }^{2}$

In practice, abnormal values of SPAP during exercise should be considered carefully due to confounding factors (i.e., age, cardiac output, and exercise protocol). The timing and magnitude of exercise-induced changes in SPAP within the exercise test protocol rather than SPAP at peak exercise, seems to contain most of the information regarding pulmonary vascular function adaptation. ${ }^{28}$ Slowly progressing increase in SPAP during exercise with development of exercise $\mathrm{PH}$ at end-stages should not necessarily be labeled as abnormal, especially in elderly patients or in those reaching age-gender predicted workload. This may be perceived as normal adaptation to exercise. ${ }^{29}$ Conversely, early and steep increases in SPAP (>60 mm Hg) at the first stages of exercise followed by a plateau often imply an advanced disease process with exercise symptoms and RV dysfunction. ${ }^{30}$

\section{LV systolic function reserve to exercise}

In MR, the LV dimensions and LVEF reflect the heart's ability to adapt to increased volume load. ${ }^{31}$ However, LV contractile function may already be impaired even in the setting of a preserved LVEF at rest. Lack of contractile reserve $(<4 \%$ increase in LVEF or a 

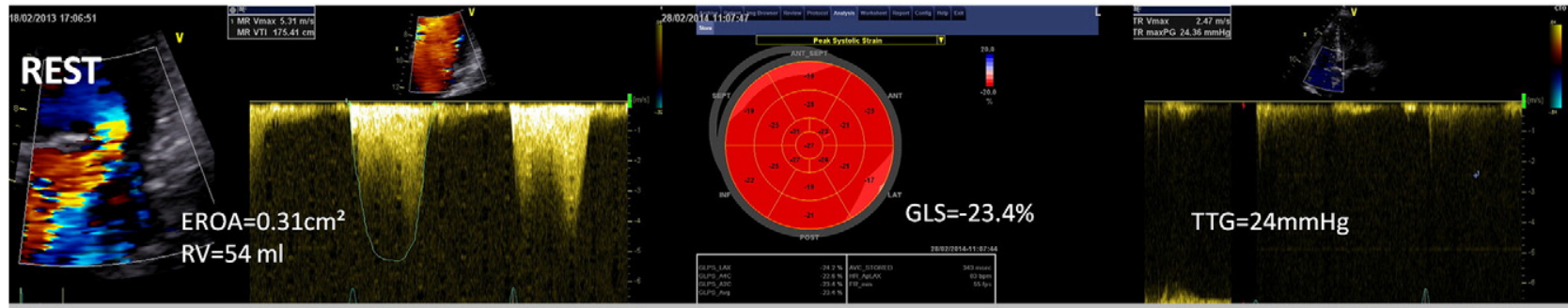

$E R O A=0.31 \mathrm{~cm}^{2}$

TTG=24mmHg

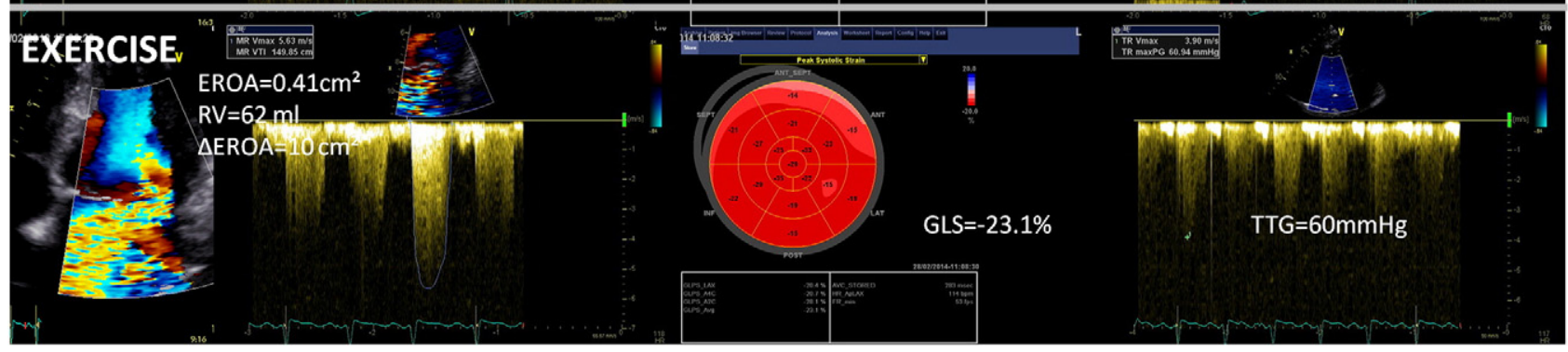

Fig. 2 - Asymptomatic female patient with moderate primary MR at rest due to posterior leaflet prolapse. There is no severe LV dilatation (end-diastolic diameter of $48 \mathrm{~mm}$, end-systolic diameter of $33 \mathrm{~mm}$ ). LV systolic function is normal, with preserved $\mathrm{LV}$ longitudinal function at rest (GLS $=-23.4 \%$ ). There is no pulmonary hypertension at rest (TTG $=24 \mathrm{~mm} \mathrm{Hg}$ ). During exercise (lower panel) MR becomes severe ( $\triangle \mathrm{EROA}=10 \mathrm{~mm}^{2}, \Delta \mathrm{RV}=8 \mathrm{ml}$ ), there is no increase in GLS and the patient develops severe pulmonary hypertension at the heart rate of $120 \mathrm{bpm}$ and at the second stage of exercise protocol (50 W). She stops the exercise test for dyspnea. MR-mitral regurgitation; LV-left ventricle; EROA-effective regurgitant orifice area; RV-regurgitant volume; GLS-global longitudinal strain by speckle tracking; TTG-transtricuspid systolic pressure gradient.

post-exercise end-systolic volume index $>25 \mathrm{~cm}^{3} / \mathrm{m}^{2}$ ) is an early marker of impaired myocyte contractility and predicts post-operative LV function after MV surgery, as well as symptom development and progressive deterioration of LV function in medically treated patients. ${ }^{31}$ Recent studies have suggested that $2 \mathrm{D}$ strain imaging obtained at rest and during exercise could be more sensitive at detecting subclinical LV dysfunction. ${ }^{11,32,33}$ A GLS below $-20 \%$ is associated with LV systolic dysfunction after MV surgery, ${ }^{31}$ and is a predictor of poorer event-free survival. ${ }^{11,33}$ Moreover, the absence of contractile reserve $(<-2 \%$ increase in global longitudinal strain during exercise) predicts postoperative LV dysfunction as well as a 2-fold increase in risk of cardiac events at follow-up (cardiovascular death, symptoms or development of LV systolic dysfunction, hospitalization for acute pulmonary edema or $\mathrm{HF}$ ) in medically-treated patients with a better sensitivity and specificity than an inadequate increase in LVEF. ${ }^{11}$ In practice, GLS using 2D speckle tracking imaging is the recommended approach; it can be successfully used during exercise, especially in the low stages when heart rate is in adequacy with the frame rate. ${ }^{11,33}$

\section{Biomarkers during exercise testing}

Brain natriuretic peptide (BNP) - a biomarker synthesized and secreted by cardiac myocytes in response to increased intracavitary pressure and wall stress, is a valuable surrogate marker of MR consequences on the LV, LA and PAP both at rest and during exercise. ${ }^{34-36} \mathrm{~A}$ recent study has shown that in asymptomatic patients with moderate or severe primary MR and normal LV dimensions and function, elevated exercise BNP was independently associated with a higher risk of cardiac events at mid-term follow-up. ${ }^{14}$ The main determinants of exercise BNP levels were resting LV filling pressures, LA enlargement and exercise induced LV longitudinal dysfunction, i.e. lower exercise GLS. An exercise BNP level $\geq 64 \mathrm{pg} / \mathrm{ml}$ was the best cut-off value to predict cardiac events. ${ }^{14}$ Further studies are needed to confirm the added value of exercise BNP in patients with primary MR.

A summary of all exercise testing derived parameters associated with poor outcome in asymptomatic patients with severe primary MR is provided in Table 2. A schematic representation of exercise testing modalities in the management of patients with primary MR is shown in Fig. 3.

\section{Secondary MR}

Chronic secondary MR (SMR) is frequently encountered in HF patients with LV systolic dysfunction of ischemic or non-ischemic etiology. ${ }^{37}$ It develops due to a combination of mitral leaflet tethering secondary to LV dilatation/deformation with papillary displacement/disco-ordination, annular dilatation/dysfunction, insufficient LV-generated closing forces due to reduction of LV contractility, and global LV/papillary muscle dyssynchrony; SMR is dynamic and affected by changes in hemodynamic conditions, which occur during exercise. Hence, ESE gives the perfect opportunity for the clinician to take a glimpse into the complexity of secondary MR in each individual patient and offers valuable clinical information.

\section{Role of ESE}

ESE is a reliable tool to assess the dynamic nature of chronic secondary MR (Fig. 4). Based on our experience, ESE may be of 


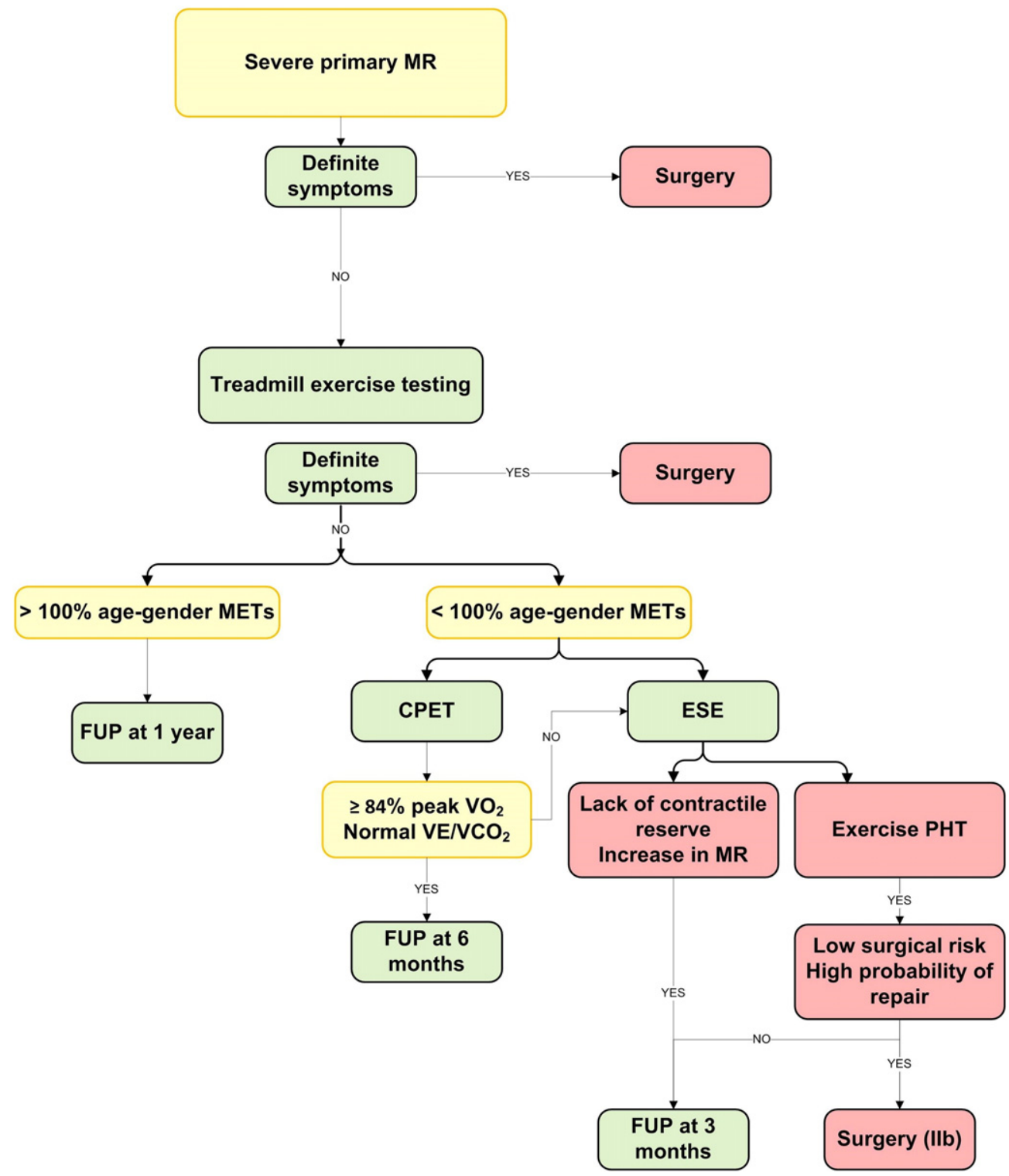

Fig. 3 - Flow-chart on the management of a patient with severe primary MR. MR-mitral regurgitation; METs-metabolic equivalents; FUP-follow-up; CPET-cardiopulmonary exercise testing; ESE-exercise stress echocardiography; peak VO2-peak oxygen consumption; VE/VCO2 the slope of the linear relationship between ventilation (VE) and carbon dioxide output (VCO2); PHT-pulmonary hypertension.

interest in the following conditions: 1) in patients with LV dysfunction who present with exertional dyspnea out of proportion to the severity of resting LV dysfunction or MR severity, 2) in patients in whom acute pulmonary edema occurs without any obvious cause; 3) to unmask patients at high risk of mortality and $\mathrm{HF}$ (increase in MR severity and $\mathrm{PH}$ with exercise); 4) before surgical revascularization in patients with moderate MR and 5) following surgery, to identify persistence of $\mathrm{PH}$ and explain the absence of functional class improvement. $^{38-40}$

The severity of SMR at rest is unrelated to the magnitude of MR changes during exercise. About $30 \%$ of patients may have a marked increase in MR with exercise accompanied by an increase in SPAP. ${ }^{41}$ The degree of exercise-induced changes in MR severity relates to changes in LV remodelling, ${ }^{42}$ in valvular deformation and a1,42 $^{\text {in }} \mathrm{LV}$ and papillary muscles synchronicity. ${ }^{43}$ Rarely, transient myocardial ischemia may lead to transient SMR. The induction of ischemia during ESE can be easily detected by new or worsening wall motion abnormalities secondary to a hemodynamically significant stenosis of the right or circumflex coronary artery. Myocardial revascularization alone may solve MR in such cases.

The magnitude of increase in MR and SPAP is more pronounced in patients with exercise-limiting dyspnea and in those hospitalized for acute pulmonary edema. ${ }^{38}$ The increase in MR degree during exercise (i.e. increase in EROA $\geq 13 \mathrm{~mm}^{2}$ ) independently predicts cardiac death and HF admission in patients with systolic heart failure and at least mild secondary ischemic MR. ${ }^{39}$ The best predictive cut-off value for the increase in SPAP associated with the outcome is 


\section{Table 2 - Parameters of poor prognosis in asymptomatic primary MR.}

\begin{tabular}{|c|c|c|}
\hline Parameter & Type of Exercise Testing & Study \\
\hline Symptom development & Any type & \\
\hline$<85 \%$ age-gender-predicted METs & Treadmill exercise testing & Naji et al. ${ }^{17,18}$ \\
\hline $\begin{array}{l}\text { Abnormal heart rate recovery ( }<18 \text { beats drop in heart rate } \\
\text { over } 1 \mathrm{~min} \text { in recovery) }\end{array}$ & Treadmill exercise testing & Naji et al. ${ }^{17,18}$ \\
\hline$\leq 84 \%$ age-gender expected peak VO2 & Cardiopulmonary exercise testing & Messika-Zeitoun et al. ${ }^{19}$ \\
\hline $\begin{array}{l}\text { Exercise induced increase in } E R O A \geq 10 \mathrm{~mm}^{2} \text { or in } \\
\text { regurgitant volume } \geq 15 \mathrm{ml}\end{array}$ & Exercise stress echocardiography & Magne et al. ${ }^{12}$ \\
\hline $\begin{array}{l}\text { Exercise induced systolic pulmonary artery } \\
\text { pressure } \geq 60 \mathrm{~mm} \mathrm{Hg}\end{array}$ & $\begin{array}{l}\text { Exercise stress echocardiography and post } \\
\text { exercise stress echocardiography }\end{array}$ & $\begin{array}{l}\text { Magne et al. }{ }^{13} \\
\text { Kusunose et al. }{ }^{24}\end{array}$ \\
\hline Exercise TAPSE $<18 \mathrm{~mm}$ & Post-exercise stress echocardiography & Kusunose et al. ${ }^{24}$ \\
\hline$<4 \%$ increase in LVEF post exercise & Post-exercise echocardiography & Lee et al. ${ }^{31}$ \\
\hline$<-2 \%$ increase in GLS during low level exercise stages & Exercise stress echocardiography & Magne et al. ${ }^{11}$ \\
\hline Exercise BNP levels $\geq 64 \mathrm{pg} / \mathrm{ml}$ & $\begin{array}{l}\text { Any type, blood sample drawn } 5 \text { min after } \\
\text { exercise cessation }\end{array}$ & Magne et al. ${ }^{14}$ \\
\hline
\end{tabular}

Peak VO2-peak oxygen consumption; EROA-effective regurgitant orifice area as assessed by PISA method; TAPSE-tricuspid annulus plane systolic excursion; LVEF-left ventricle ejection fraction; LV-left ventricle; GLS-global longitudinal strain as assessed by speckle tracking analysis; BNP-brain natriuretic peptide.

$21 \mathrm{~mm} \mathrm{Hg}$. Exercise induced PH (i.e. SPAP $>60 \mathrm{~mm} \mathrm{Hg}$ ) is common in secondary MR patients, up to $40 \%$ of patients in one study (Fig. 4). ${ }^{40}$ It seems to be related more to exercise MR severity and LV diastolic function than to NYHA class. Moreover, exercise $\mathrm{PH}$ has proven to be a powerful predictor of the occurrence of HF decompensation, cardiac-related death, or combined cardiovascular events in these patients. ${ }^{40}$ The presence of viable myocardium predicts LV reverse remodelling and a better outcome after revascularization, beta-blocker therapy and cardiac resynchronization therapy CRT. ${ }^{44}$ LV reverse remodelling and reduced mitral apparatus deformation after CRT are associated with a reduction in both resting and exercise-induced MR and an improvement in cardiopulmonary performance. ${ }^{45}$ Moreover, SMR decreases more significantly in patients with viability in the region of the pacing lead after CRT. ${ }^{46}$

Considering the adverse prognostic implications of the increase in MR severity with exercise in patients with moderate SMR, the ESC guidelines suggested combined MV repair at the time of surgical coronary revascularization in patients in whom such a response has been demonstrated with ESE (Class IIa).

\section{Conclusions}

Exercise testing, in its many forms, is useful for the evaluation of asymptomatic patients with primary MR. It allows symptom assessment, confident link of symptoms to VHD severity (ESE, CPET), safe deferral of surgery for the next 1-year if the patient proves to have a preserved exercise capacity, insights into the mechanism of exercise induced dyspnea (CPET and ESE) and helps in risk stratification of truly asymptomatic patients through the assessment of exercise-induced $\mathrm{PH}, \mathrm{LV}$ dysfunction and MR increase in severity. For all these reasons, exercise testing should be encouraged in asymptomatic patients with primary MR. Moreover, ESE is useful in the evaluation of patients with ischemic SMR for risk stratification as well as for the detection of those with moderate ischemic MR in whom MV repair at the time of surgical

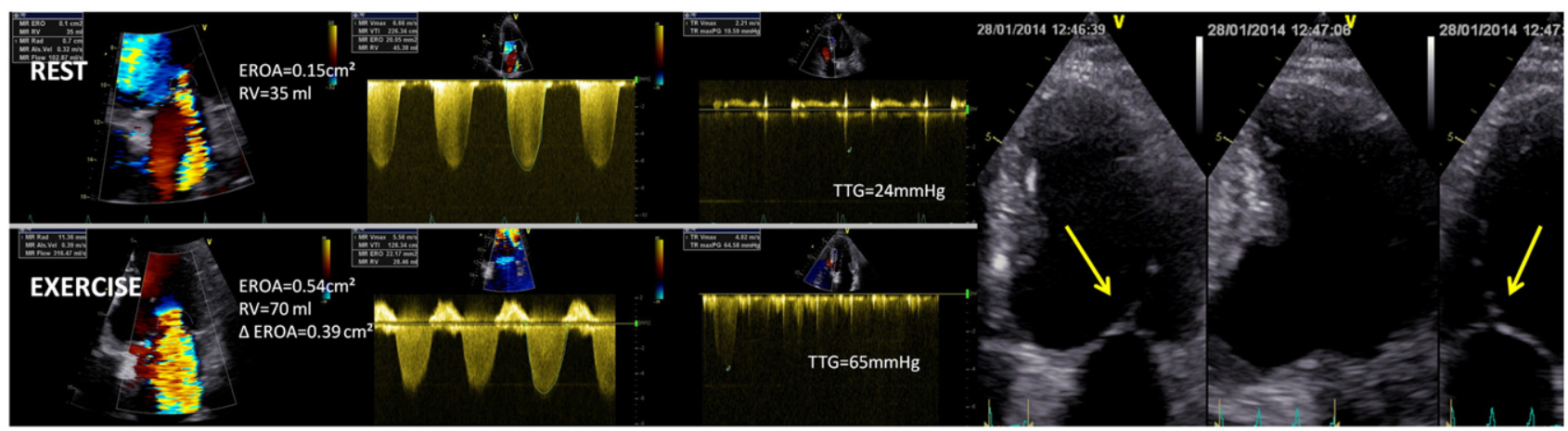

Fig. 4 - High-risk patient with secondary ischemic MR. At rest, upper panel, there is moderate ischemic MR, important tethering on the mitral valve leaflets (yellow arrows) and no pulmonary hypertension. During exercise (lower panel), there is a severe increase in MR (exercise induces change in EROA of $0.39 \mathrm{~cm}^{2}$ ) accompanied by pulmonary hypertension and dyspnea. This is a high-risk patient for future heart failure episodes or mortality. MR-mitral regurgitation; EROA-effective regurgitant orifice area; RV-regurgitant volume; TTG-transtricuspid systolic pressure gradient. 
revascularization may add benefit. The role of ESE in non-ischemic forms of secondary MR is not yet established and should be further explored.

\section{Statement of conflict of interest}

There is no conflict of interest of any of the listed authors.

\section{REFEREN C ES}

1. Iung B, Baron G, Butchart EG, et al. A prospective survey of patients with valvular heart disease in Europe: the Euro heart survey on valvular heart disease. Eur Heart J. 2003;24: 1231-1243.

2. Vahanian A, Alfieri O, Andreotti F, et al. Guidelines on the management of valvular heart disease (version 2012). Eur Heart J. 2012;33:2451-2496.

3. Nishimura RA, Otto CM, Bonow RO, et al. 2017 AHA/ACC focused update of the 2014 AHA/ACC guideline for the management of patients with valvular heart disease: a report of the American College of Cardiology/American Heart Association Task Force on Clinical Practice Guidelines. J Am Coll Cardiol. 2017;70:252-289.

4. Enriquez-Sarano M, Avierinos JF, Messika-Zeitoun D, et al. Quantitative determinants of the outcome of asymptomatic mitral regurgitation. N Engl J Med. 2005;352:875-883.

5. Detaint D, Sundt TM, Nkomo VT, et al. Surgical correction of mitral regurgitation in the elderly: outcomes and recent improvements. Circulation. 2006;114:265-272.

6. Feldman T, Kar S, Elmariah S, et al. Randomized comparison of percutaneous repair and surgery for mitral regurgitation: 5-year results of EVEREST II. J Am Coll Cardiol. 2015;66: 2844-2854.

7. Nishimura RA, Otto CM, Bonow RO, et al. 2014 AHA/ACC guideline for the management of patients with valvular heart disease: executive summary: a report of the American College of Cardiology/American Heart Association Task Force on Practice Guidelines. J Am Coll Cardiol. 2014;63:2438-2488.

8. Rusinaru D, Tribouilloy C, Grigioni F, et al. Left atrial size is a potent predictor of mortality in mitral regurgitation due to flail leaflets: results from a large international multicenter study. Circ Cardiovasc Imaging. 2011;4:473-481.

9. Barbieri A, Bursi F, Grigioni F, et al. Prognostic and therapeutic implications of pulmonary hypertension complicating degenerative mitral regurgitation due to flail leaflet: a multicenter long-term international study. Eur Heart J. 2011;32: 751-759.

10. Eguchi K, Ohtaki E, Matsumura T, et al. Pre-operative atrial fibrillation as the key determinant of outcome of mitral valve repair for degenerative mitral regurgitation. Eur Heart $\mathrm{J}$. 2005;26:1866-1872.

11. Magne J, Mahjoub H, Dulgheru R, et al. Left ventricular contractile reserve in asymptomatic primary mitral regurgitation. Eur Heart J. 2014;35:1608-1616.

12. Magne J, Lancellotti P, Pierard LA. Exercise-induced changes in degenerative mitral regurgitation. J Am Coll Cardiol. 2010;56: 300-309.

13. Magne J, Donal E, Mahjoub H, et al. Impact of exercise pulmonary hypertension on postoperative outcome in primary mitral regurgitation. Heart. 2015;101:391-396.

14. Magne J, Mahjoub H, Pibarot P, et al. Prognostic importance of exercise brain natriuretic peptide in asymptomatic degenerative mitral regurgitation. Eur J Heart Fail. 2012;14:1293-1302.
15. Magne J, Lancellotti P, Pierard LA. Exercise pulmonary hypertension in asymptomatic degenerative mitral regurgitation. Circulation. 2010;122:33-41.

16. Suzuki $\mathrm{K}$, Izumo $\mathrm{M}$, Yoneyama $\mathrm{K}$, et al. Influence of exercise-induced pulmonary hypertension on exercise capacity in asymptomatic degenerative mitral regurgitation. $J$ Cardiol. 2015;66:246-252.

17. Naji P, Griffin BP, Asfahan F, et al. Predictors of long-term outcomes in patients with significant myxomatous mitral regurgitation undergoing exercise echocardiography. Circulation. 2014;129:1310-1319.

18. Naji P, Griffin BP, Barr T, et al. Importance of exercise capacity in predicting outcomes and determining optimal timing of surgery in significant primary mitral regurgitation. J Am Heart Assoc. 2014;3:e001010.

19. Messika-Zeitoun D, Johnson BD, Nkomo V, et al. Cardiopulmonary exercise testing determination of functional capacity in mitral regurgitation: physiologic and outcome implications. J Am Coll Cardiol. 2006;47:2521-2527.

20. Lancellotti P, Pellikka PA, Budts W, et al. The clinical use of stress echocardiography in non-ischaemic heart disease: recommendations from the European Association of Cardiovascular Imaging and the American Society of Echocardiography. Eur Heart J Cardiovasc Imaging. 2016;17:1191-1229.

21. Guazzi M, Arena R, Halle M, et al. 2016 focused update: clinical recommendations for cardiopulmonary exercise testing data assessment in specific patient populations. Eur Heart J. 2016, https://doi.org/10.1093/eurheartj/ehw180. [pii: ehw180. Epub ahead of print].

22. Guazzi M, Cahalin LP, Arena R. Cardiopulmonary exercise testing as a diagnostic tool for the detection of left-sided pulmonary hypertension in heart failure. J Card Fail. 2013;19: 461-467.

23. Coisne A, Levy F, Malaquin D, et al. Feasibility of Doppler hemodynamic evaluation of primary and secondary mitral regurgitation during exercise echocardiography. Int $J$ Cardiovasc Imaging. 2015;31:291-299.

24. Kusunose K, Popovic ZB, Motoki H, Marwick TH. Prognostic significance of exercise-induced right ventricular dysfunction in asymptomatic degenerative mitral regurgitation. Circ Cardiovasc Imaging. 2013;6:167-176.

25. Lee R, Hanekom L, Marwick TH, Leano R, Wahi S. Prediction of subclinical left ventricular dysfunction with strain rate imaging in patients with asymptomatic severe mitral regurgitation. Am J Cardiol. 2004;94:1333-1337.

26. Naji P, Asfahan F, Barr T, et al. Impact of duration of mitral regurgitation on outcomes in asymptomatic patients with myxomatous mitral valve undergoing exercise stress echocardiography. J Am Heart Assoc. 2015;4, https://doi.org/ 10.1161/JAHA.114.001348. [pii:e001348].

27. Magne J, Lancellotti P, O'Connor K, et al. Prediction of exercise pulmonary hypertension in asymptomatic degenerative mitral regurgitation. J Am Soc Echocardiogr. 2011;24:1004-1012.

28. Tolle JJ, Waxman AB, Van Horn TL, Pappagianopoulos PP, Systrom DM. Exercise-induced pulmonary arterial hypertension. Circulation. 2008;118:2183-2189.

29. Mahjoub H, Levy F, Cassol M, et al. Effects of age on pulmonary artery systolic pressure at rest and during exercise in normal adults. Eur J Echocardiogr. 2009;10:635-640.

30. Lewis GD, Murphy RM, Shah RV, et al. Pulmonary vascular response patterns during exercise in left ventricular systolic dysfunction predict exercise capacity and outcomes. Circ Heart Fail. 2011;4:276-285.

31. Lee R, Haluska B, Leung DY, et al. Functional and prognostic implications of left ventricular contractile reserve in patients with asymptomatic severe mitral regurgitation. Heart. 2005;91:1407-1412. 
32. Haluska BA, Short L, Marwick TH. Relationship of ventricular longitudinal function to contractile reserve in patients with mitral regurgitation. Am Heart J. 2003;146:183-188.

33. Donal E, Mascle S, Brunet A, et al. Prediction of left ventricular ejection fraction 6 months after surgical correction of organic mitral regurgitation: the value of exercise echocardiography and deformation imaging. Eur Heart J Cardiovasc Imaging. 2012;13:922-930.

34. Detaint D, Messika-Zeitoun D, Avierinos JF, et al. B-type natriuretic peptide in organic mitral regurgitation: determinants and impact on outcome. Circulation. 2005;111:2391-2397.

35. Kerr AJ, Raffel OC, Whalley GA, Zeng I, Stewart RA. Elevated B-type natriuretic peptide despite normal left ventricular function on rest and exercise stress echocardiography in mitral regurgitation. Eur Heart J. 2008;29:363-370.

36. Klaar U, Gabriel H, Bergler-Klein J, et al. Prognostic value of serial B-type natriuretic peptide measurement in asymptomatic organic mitral regurgitation. Eur J Heart Fail. 2011;13:163-169.

37. Rossi A, Dini F, Faggiano P, et al. Independent prognostic value of functional mitral regurgitation in patients with heart failure. A quantitative analysis of 1256 patients with ischaemic and non-ischaemic dilated cardiomyopathy. Heart. 2011;97:1675-1680.

38. Pierard LA, Lancellotti P. The role of ischemic mitral regurgitation in the pathogenesis of acute pulmonary edema. $\mathrm{N}$ Engl $\mathrm{J}$ Med. 2004;351:1627-1634.

39. Lancellotti P, Gerard PL, Pierard LA. Long-term outcome of patients with heart failure and dynamic functional mitral regurgitation. Eur Heart J. 2005;26:1528-1532.
40. Lancellotti P, Magne J, Dulgheru R, et al. Clinical significance of exercise pulmonary hypertension in secondary mitral regurgitation. Am J Cardiol. 2015;115:1454-1461.

41. Lancellotti P, Lebrun F, Pierard LA. Determinants of exercise-induced changes in mitral regurgitation in patients with coronary artery disease and left ventricular dysfunction. J Am Coll Cardiol. 2003;42:1921-1928.

42. Giga V, Ostojic M, Vujisic-Tesic B, et al. Exercise-induced changes in mitral regurgitation in patients with prior myocardial infarction and left ventricular dysfunction: relation to mitral deformation and left ventricular function and shape. Eur Heart J. 2005;26:1860-1865.

43. Lancellotti P, Pierard LA. Chronic ischaemic mitral regurgitation: exercise testing reveals its dynamic component. Eur Heart J. 2005;26:1816-1817.

44. Moonen M, O'Connor K, Magne J, Lancellotti P, Pierard LA. Stress echocardiography for selecting potential responders to cardiac resynchronisation therapy. Heart. 2010;96:1142-1146.

45. Madaric J, Vanderheyden M, Van Laethem C, et al. Early and late effects of cardiac resynchronization therapy on exercise-induced mitral regurgitation: relationship with left ventricular dyssynchrony, remodelling and cardiopulmonary performance. Eur Heart J. 2007;28:2134-2141.

46. Senechal M, Lancellotti P, Magne J, et al. Impact of mitral regurgitation and myocardial viability on left ventricular reverse remodeling after cardiac resynchronization therapy in patients with ischemic cardiomyopathy. Am J Cardiol. 2010;106:31-37. 